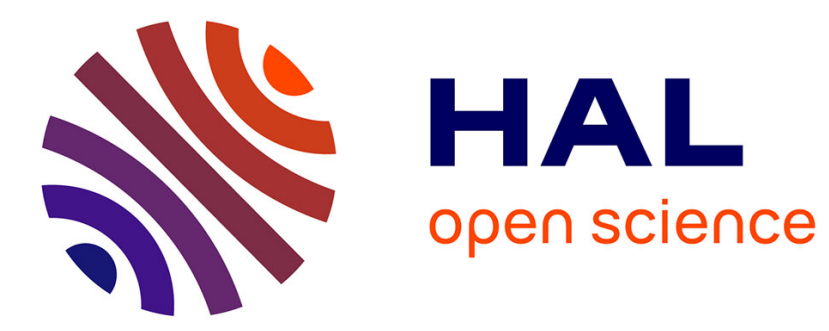

\title{
Modulation of parafoveal word processing by cognitive load during modified visual search tasks
}

Julien Dampuré, Abdelrhani Benraiss, Nicolas Vibert

\section{To cite this version:}

Julien Dampuré, Abdelrhani Benraiss, Nicolas Vibert. Modulation of parafoveal word processing by cognitive load during modified visual search tasks. Quarterly Journal of Experimental Psychology, 2019, 72, pp.1805-1826. 10.1177/1747021818811123 . hal-02109322

\section{HAL Id: hal-02109322 \\ https://hal.science/hal-02109322}

Submitted on 29 Dec 2020

HAL is a multi-disciplinary open access archive for the deposit and dissemination of scientific research documents, whether they are published or not. The documents may come from teaching and research institutions in France or abroad, or from public or private research centers.
L'archive ouverte pluridisciplinaire HAL, est destinée au dépôt et à la diffusion de documents scientifiques de niveau recherche, publiés ou non, émanant des établissements d'enseignement et de recherche français ou étrangers, des laboratoires publics ou privés. 
23 Address for correspondence:

24 Julien Dampuré, PhD, Cognitive Neuroscience and Psycholinguistics lab, Institute for 25 Biomedical Technologies (IBT), University of La Laguna, La Laguna 38205, Spain. modified visual search tasks

\section{Julien DAMPURE ${ }^{1,2}$ (corresponding author)}

Phone: + 34 922317515, julien.dampure@ hotmail.fr

\section{Abdelrhani BENRAISS ${ }^{1}$} Phone: + 33 (0)549454127, abdelrhani.benraiss@ univ-poitiers.fr

\section{Nicolas VIBERT ${ }^{1}$}

Phone: + 33 (0)549454634, nicolas.vibert@univ-poitiers.fr

${ }^{\mathbf{1}}$ Centre de Recherches sur la Cognition et l'Apprentissage; CNRS; Université de Poitiers; Université François Rabelais de Tours; F-86000 Poitiers, France.

${ }^{2}$ Cognitive Neuroscience and Psycholinguistics lab, Institute for Biomedical Technologies (IBT), University of La Laguna, La Laguna 38205, Spain.

\section{Modulation of parafoveal word processing by cognitive load during}


Abstract

2 electroencephalography, reading processed in visual search contexts.

During visual search for simple items, the amount of information that can be processed in parafoveal vision depends on the cognitive resources that are available. However, whether this applies to the semantic processing of words remains controversial. This work was designed to manipulate simultaneously two sources of cognitive load in order to study their impact on the depth of parafoveal word processing during a modified visual search task. The participants had to search for target words among parafoveally presented semantic, orthographic or target-unrelated distractor words while their electroencephalogram was recorded. The task-related load was manipulated by either giving target words in advance (literal task) or giving only a semantic clue to define them (categorical task). The foveal load was manipulated by displaying either a word or hash symbols at the center of the screen. Parafoveal orthographic and semantic distractors had an impact on the early event-related potential component $\mathrm{P} 2 \mathrm{a}$ only in the literal task and when hash symbols were displayed at the fovea, i.e. when both the task-related and foveal loads were low. The data show that all sources of cognitive load must be considered to understand how parafoveal words are 
The parafovea is the part of the visual field surrounding the fovea that extends from

2 approximately 2 to 5 degrees of visual angle. It is involved in information pre-processing, attention guidance and saccade programming during various visual activities such as scene exploration, visual search and reading (Rayner, 2009). Parafoveal processing has been well studied in the context of visual search tasks, for which several models have been designed (Deco \& Zihl, 2006; Desimone \& Duncan, 1995; Wolfe, 2007; Zelinsky, 2008). However, the extension of these models of visual search to more abstract stimuli such as words has been questioned (Léger, Rouet, Ros, \& Vibert, 2012; Dampuré, Ros, Rouet, \& Vibert, 2014). More generally, the extent to which the cognitive system is able to quickly access and use the meaning of words presented in parafoveal vision during complex tasks such as visual search or reading is a matter of debate.

Studies on event-related potentials (ERP) suggest that the meaning of words can be processed rapidly in parafoveal vision. Indeed, words presented in parafoveal vision can modulate ERP components related to semantic processing in reading tasks such as lexical decision (Coulson, Federmeier, Van Petten, \& Kutas, 2005; Cristescu \& Nobre, 2008) or the sequential reading of briefly presented groups of words (Barber, Ben-zvi, Bentin, \& Kutas, 2011; Barber, Duñamayor, Kutas, \& Münte, 2010; Barber, Van der Meij, \& Kutas, 2013; Stites, Payne, \& Federmeier, 2017). Several authors were able to observe semantic parafoveal preview benefits during normal reading both in Chinese (Li, Niefind, Wang, Sommer, \& Dimigen, 2015; Zhang, Li, Wang, \& Wang, 2015; Yan, Richter, Shu, and Kliegl, 2009) and in 21 alphabetic languages (López-Peréz, Dampuré, Hernández-Cabrera, \& Barber, 2016; 22 Hohenstein \& Kliegl, 2014; Schotter, 2013). Whether parafoveally previewed words are semantically processed during the reading of alphabetic languages would depend on several factors. They include the depth of orthography of the language, the capitalization of the words' first letters, the degree of similarity between preview and target, the predictability of 
1 the parafoveal word, but also the current foveal processing load and the amount of attentional

2 resources that are available (Barber et al., 2013; Rayner, Schotter, \& Drieghe, 2014; Schotter,

3 2013; Stites et al., 2017). Accordingly, Payne, Stites, and Federmeier (2016) recently

4 demonstrated that manipulating the amount of cognitive resources needed to process the

5 foveal word had an impact on the depth of parafoveal word processing during reading (see

6 also Veldre \& Andrews, 2015).

To the best of our knowledge, no ERP study has investigated how parafoveal word processing is performed during visual search for words. In this article, we claim that in visual search as during reading, the depth of parafoveal word processing depends on the amount of cognitive resources that are, first, necessary and, second, available to access high-level word representations. In particular, parafoveal semantic processing may depend on (1) how much the features of the parafoveal word are already activated in memory and (2) the different sources of cognitive load associated with the visual search process.

\section{Influence of the activated elements in memory on parafoveal processing}

Models of visual search contend that the items that match the visual or semantic features of the target are fixated more often than target-unrelated stimuli (Harris \& Pashler, 2004; Huettig, Olivers, \& Hartsuiker, 2011; Moores, Laiti, \& Chelazzi, 2003; Olivers, Meijer,

\& Theeuwes, 2006; Soto \& Humphreys, 2007). The target template kept in working memory

21 guides the searcher's attention towards the object (Downing, 2000; Harris \& Pashler, 2004;

22 Olivers et al., 2006; Soto \& Humphreys, 2007; Soto, Heinke, Humphreys, \& Blanco, 2005;

23 Wolfe, Horowitz, Kenner, Hyle, \& Vasan, 2004) or picture (Moores et al., 2003; Telling,

24 Kumar, Meyer, \& Humphreys, 2010) that has the most features in common with the target.

25 Then, a saccade is triggered toward the selected item, and a recognition process takes place in 
1 order to categorize it as the target or a distractor. At that stage, the distractor items that share

2 features with the target are often fixated for longer durations than unrelated distractors

3 because more time is needed to distinguish them from the target (Becker, 2011; Belke,

4 Humphreys, Watson, Meyer, \& Telling, 2008; Meyer, Belke, Telling, \& Humphreys, 2007;

5 Potts, Liotti, Tucker, \& Posner, 1996). This alternation between early selection and late

6 rejection mechanisms goes on until the target is found (Zelinsky, 2008).

Similarly, word processing studies report that people's attention is guided toward the words in the parafoveal visual field that share features with the content of their working memory (Dampuré et al., 2014; Huettig \& McQueen, 2007; Huettig et al., 2011; Léger \& Tijus, 2007; Léger et al., 2012). During visual search for words, the searcher's gaze is 11 preferentially attracted by words that look like the target word, but also by words that are 12 semantically related to the target word (Léger et al., 2012; Dampuré et al., 2014). Hence, fast lexico-semantic matching between the target template and a semantically-related parafoveal word can occur and is sufficiently efficient to guide the participant's attention. The most likely explanation is that learning the target word before searching for it pre-activates both the meaning and visual form of visually similar words (Boot \& Pecher, 2008; Grainger \& Jacobs, 1996; Rodd, 2004), and pre-activates the lexical representations of the semantic associates of the target word through spreading activation (Heil, Rolke, \& Pecchinenda, 2004; Neely, 1977). These data suggest that whether or not the meaning of a word seen in parafoveal vision can be grasped depends on the level of pre-activation of the word's representation in memory. According to the ERP literature on visual search for objects or pictures, the comparison between what is perceived and the template stored in working memory is reflected by an early positive ERP component recorded over the frontal scalp regions, the P2 or P2a component (Luck \& Hillyard, 1994; Potts et al., 1996; Potts \& Tucker, 2001; Potts, 
1 similarity between the target and the stimuli perceived in either the foveal or the

2 parafoveal/peripheral visual field. Recently, Dampuré, Benraiss, and Vibert (2016)

3 demonstrated that during visual search for target words within words presented in the foveal

4 field, the P2a component was also modulated by both visual and semantic similarity of

5 distractors to the target word. In addition, the degree of similarity between the distractors and

6 the target modulated two late ERP components that have been related to word semantic

7 processing. The frontal N300 component would reflect early automatic processing of

8 semantic similarities and/or category membership (Cristescu \& Nobre, 2008; Franklin, Dien,

9 Neely, Huber, \& Waterson, 2007; Ruz, Madrid, Lupiañez, \& Tudela, 2003). The centro-

10 parietal N400 component (Barber et al., 2013; Coulson et al., 2005; Franklin et al., 2007;

11 Kutas \& Federmeier, 2011) would reflect the automatic and/or expectation-based spreading

12 activation processes taking place in the mental lexicon.

Altogether, the degree of activation in memory of the visual and semantic features of

14 parafoveal words would determine the amount of cognitive resources needed to process them.

15 This implies that the efficiency of parafoveal processing depends on the overall quantity of 16 the cognitive resources available. The next section reviews the main sources of variations of 17 cognitive load that may interfere with parafoveal processing and interact with the degree of 18 activation of the parafoveal words in memory.

\section{Influence of various types of cognitive load on attention guidance}

Since, as stated above, working memory plays a central role in attention guidance, any load imposed on working memory should decrease the efficiency of attention guidance mechanisms and parafoveal processing during visual search for words. This idea has actually 
1 Fockert, 2005), whereby two resource-dependent mechanisms would determine the extent to

2 which a stimulus can be perceived and processed in peripheral vision.

The first one is the perceptual selection mechanism, which is a passive, automated selection system that favors foveal processing and will process parafoveal stimuli only if the amount of information to process does not exceed the current capacities. In other words, parafoveal stimuli will be processed only if the foveal perceptual load is low. The mere presence of a stimulus at the fovea is enough to deplete peripheral processing capacities, even when the participants are instructed to ignore the foveal information (Holmes, Cohen, Haith, \& Morrison, 1977). In the context of reading, this phenomenon is referred to as the effect of "foveal load" on parafoveal word processing (Henderson \& Ferreira, 1990; Hohenstein \& Kliegl, 2014; Schroyens, Vitu, Brysbaert, \& d'Ydewalle, 1999), namely the impact of the load associated with the processing of the fixated word on the processing of the word located just on the right of the fixated word. As expressed in Lavie's Load Theory, an increased foveal load would decrease the amount of cognitive resources available for parafoveal word processing (Williams, 1988) and reduce the parafoveal preview effect in reading (for a review, see Rayner, 2009). Accordingly, several word processing studies demonstrated that parafoveal semantic processing can occur when no information must be processed in foveal vision and all cognitive resources can be allocated to parafoveal processing (Calvo \& Castillo, 2009; Cristescu \& Nobre, 2008), or when time constraints are sufficiently low to allow simultaneous processing of both the foveal and parafoveal words (Barber et al., 2013). The second mechanism is a late, active selection mechanism controlled by executive functions. The Load Theory emphasizes the role of working memory, which determines the priorities used to orient processing toward the task-relevant stimuli and avoid the processing of task-irrelevant stimuli (Lavie, Hirst, Fockert, \& Viding, 2004). This is highly reminiscent of the role that working memory fulfills in visual search models by keeping in mind the 
1 target-template in order to guide attention toward the relevant stimuli. Consequently, a high 2 working memory load should increase distractibility or, in other words, decrease the 3 efficiency of attentional selection. Accordingly, various studies have demonstrated that the

4 visual span, i.e., the region of the visual field from which information can be extracted in a 5 glance (see Rayner, 1998 for a review) decreases when another task must be performed 6 simultaneously (in dual-task paradigms) or when the difficulty of the task increases 7 (Kornrumpf \& Sommer, 2015; Pomplun, Reingold, \& Shen, 2001). Hence, in the context of visual search, the ability to guide the attention toward relevant, template-matching stimuli also decreases when the working memory load imposed by the task increases (Desimone \& Duncan, 1995; Duncan \& Humphreys, 1989; Zhang, Zhang, Huang, Kong, \& Wang, 2011). Altogether, evidence points toward a simultaneous influence of both the foveal load and working memory load on attentional guidance during visual search. Indeed, in the context of visual search for words, Dampuré et al. (2014) demonstrated for example that the impact of distractor words, which were related either orthographically or semantically to the target word, on attention guidance depended on the nature of the search task. The participants had to search for a target word that was either given before each trial (literal task, Experiment 1) or defined only by its semantic category (categorical search, Experiment 2). The results showed that the searchers' gaze was more attracted by both kinds of distractor words in the literal task than in the categorical task. The categorical task was more difficult, as shown by the time needed to find target words, which was roughly twice longer in the categorical task than in the 21 literal task.

From a cognitive point of view, the categorical task has been associated with a higher working memory load because the target template is less well defined than in the literal task, since the participants do not know with certainty which word they are searching for (Schmidt, MacNamara, Proudfit, \& Zelinsky, 2014). Indeed, the ability to create and to maintain a 
1 stable, well-defined target template in working memory was proven to have a strong impact 2 on attention guidance (Hout \& Goldinger, 2015; Wolfe, Butcher, Lee, \& Hyle, 2003; Wolfe et

3 al., 2004). In addition, the comparison between the target template and the words perceived in

4 the peripheral visual field requires more cognitive resources in the categorical task because

5 the searcher must access the meaning of each word in the display to decide whether it is the 6 target word, which is not necessary in the literal task.

Additionally, Dampuré et al. (2014) demonstrated that within each task, the efficiency of attention guidance depended on the amount of concomitant foveal processing that was needed. In the categorical task for instance, participants' gaze was attracted by distractor words that were related to the target word only at the beginning of the search phase, when the

11 participants were not fixating any word, but not later on in the search phase when they were 12 fixating words in foveal vision.

\section{Rationale of the experiment and hypotheses}

According to the previous sections, the efficiency of parafoveal word processing depends on (1) the working memory load imposed by the task and (2) within each task, the load generated by the current foveal processing. To the best of our knowledge, the present ERP study is the first to investigate the simultaneous impact of both the foveal and working memory loads on the attentional selection of parafoveal words during visual search.

With this goal in mind, we modified the experimental design used by Dampuré et al. (2016), in which the participants had to search for a target word among distractor words presented one by one at the center of the screen. The distractor words included "unrelated distractors" that were unrelated to the target word, "orthographic distractors" that looked like the target word and "semantic distractors" that were semantically related to the target word. In 
1 the present study, the same words (i.e. the target words and orthographic, semantic and target-

2 unrelated distractors) were presented in parafoveal vision, at five degrees of visual angle from

3 the center of the screen, in order to test the efficiency of parafoveal processing.

The working memory load was manipulated by varying the nature of the search task.

As in Dampuré et al. (2016), participants were instructed to search for target words that either were given in advance (literal task) or defined by their super-ordinate category (categorical task). Because the categorical task generates a higher working memory load than the literal one (Dampuré et al., 2014, 2016; Schmidt et al., 2014; Hout \& Goldinger, 2015), more cognitive resources should be available for parafoveal processing in the literal task. Concomitantly, the foveal load was manipulated in both the literal and categorical tasks by varying the nature of the stimulus displayed at the center of the screen (i.e., on the participants' fovea) while the parafoveal words were processed. The foveal stimulus could be either a series of hash symbols or a word unrelated to the target. Since the words were more task-relevant and would be automatically processed (Holmes et al., 1977) even though the participants were instructed not to pay any attention to what appeared in their foveal vision, the foveal load was expected to be higher with the word than with task-irrelevant hash symbols repeated across the entire experiment (Lavie \& Robertson, 2001). Hence, more cognitive resources should be available for parafoveal processing when a word rather than hash symbols is present at the center of the screen.

The two first hypotheses stated therefore that parafoveal processing should be slower and less accurate in the categorical task than in the literal task (hypothesis 1), and also slower and less accurate when the foveal stimulus is a word rather than hash symbols (hypothesis 2). As a result, the impact of parafoveal orthographic and semantic distractors on error rates and response times compared to parafoveal target-unrelated distractors should be stronger in the literal task than in the categorical task and stronger when the foveal stimulus is hash symbols 
1 rather than a word. In addition, in accordance with earlier studies of visual search for words

2 (Dampuré et al., 2014, 2016; Léger et al., 2012) a third hypothesis stated that orthographic

3 and semantic distractors should have a differential impact on error rates and response times,

4 and that this impact should also vary according to the nature of the search task.

Regarding the ERP responses, Dampuré et al. (2016) reported modulations of the P2a,

6 N300 and N400 ERP components by orthographic and semantic distractors presented at the

7 center of the screen compared with unrelated distractors. The same three ERP components were targeted in the present experiment in order to be able to compare data from the present experiment with those reported in et al.'s paper. However, because the present experiment was designed to reproduce the parafoveal processing that leads to decide toward which word

11 in the visual field the next saccade will be done during visual search, the participants did not have to identify the parafoveal word, but just to tell whether they believed or not it could be the target. Since in visual search situations, this decision is generally taken within less than 400 milliseconds (Dampuré et al., 2014; Léger et al., 2012), the parafoveal orthographic and/or semantic distractors were expected to modulate mostly the frontal P2a and N300 components compared to parafoveal unrelated distractors.

As stated above, more cognitive resources should be available for parafoveal processing when both the task-related working memory load and the foveal load are low. The fourth hypothesis stated therefore that the strongest modulation of the P2a and N300 components by parafoveal orthographic and/or semantic distractors compared to parafoveal unrelated distractors was expected in the literal task and with hash symbols at the center of the screen. Either performing the categorical task or gazing at a word instead of hash symbols was expected to reduce the impact of parafoveal distractors. The parafoveal target-related distractors were expected to have even less or no impact when both the task-related working memory load and the foveal load were high, i.e., in the categorical task and with a word at the 
1 center of the screen. Consequently, the modulations of the P2a and N300 components by

2 parafoveal orthographic and/or semantic distractors compared to target-unrelated distractors

3 were expected to be strongly reduced or to disappear in these conditions. As for error rates

4 and response times, an additional fifth hypothesis stated that orthographic and semantic

5 distractors should have a differential impact on ERP components, and that this impact should

6 vary according to the nature of the search task. Altogether, according to hypotheses 4 and 5 ,

7 the modulations of the P2a and N300 components should result from four-way interactions

8 between the nature of the task, the nature of the foveal stimulus, the target-relatedness

9 condition (target-related distractors versus target-unrelated distractors) and the type of

10 distractor (orthographic or semantic distractors).

11 Finally, a set of experimental displays in which two orthographic or two semantic

12 distractors were displayed simultaneously in foveal and parafoveal vision (see the method 13 section below) was included in the experiment. They were designed to compare the

14 modulations of the ERP components by foveal orthographic and/or semantic distractors 15 compared to foveal unrelated distractors obtained as the participants concentrated on the 16 parafoveal words with the modulations reported by Dampuré et al. (2016) in response to the 17 same foveal distractors presented in isolation. The goal was to ensure that the participants 18 were processing the stimulus appearing at the fovea even when asked to concentrate on the 19 parafoveal words.

\section{Participants}


Eighteen psychology students ( 8 women) from the University of Poitiers (France)

2 participated in this experiment. They were all native French speakers, right-handed, with normal or corrected-to-normal vision and were 18 to 33 years old $(M=23$ years, $S D=4)$. The students were all volunteers and provided their written informed consent before the study.

Apparatus

7

All words or symbols were displayed using the E-Prime 1.0 software on a 21-inch screen (resolution 1024 x 768 pixels), in white on a black background and in lowercase 14point Calibri font. Participants sat comfortably at $60 \mathrm{~cm}$ from the screen in a shielded, air-

11 conditioned recording room. Since the human fovea covers about $2^{\circ}$ of visual angle (Jacob \& Hochstein, 2009), the foveal visual field corresponded to a $21 \mathrm{~mm}$ diameter circle on the screen. The electroencephalographic (EEG) signal was collected using an elastic cap containing 64 electrodes arranged in the international 10-20 system and connected to the NeuroScan acquisition system (Compumedics Neuroscan, Abbotsford, Victoria, Australia).

\section{Material}

The experiment included 46 "search trials" (24 experimental search trials and 22 filler search trials) during which participants had to search each time for a particular target word. Half of the search trials took the form of a literal search where the target word was given in advance, whereas the other half of the trials corresponded to categorical searches where the target word was defined by its super-ordinate category.

A search trial consisted in finding the target word among a series of parafoveal distractor words presented one by one on successive "search displays" (see Figure 1A). Each 
1 search display showed a single word within the participants' parafoveal vision, i.e., five

2 centimeters down from the center of the screen (the "parafoveal word"), and either a series of

3 hash symbols or another word at the center of the screen in the participants' foveal vision (the

4 "foveal stimulus"). Five centimeters represented 4.76 degrees of angle at the viewing distance

5 of 60 centimeters. For each search display, participants were asked to tell whether they

6 believed the parafoveal word might be the word they were looking for or not (see procedure

7 below and Figure 1B) up until the target word appeared.

The same words as those used by Dampuré et al. $(2014,2016)$ were used in this experiment. All the words were French common nouns that contained 2 to 4 syllables, had 4 to 10 letters $(\mathrm{M}=7.2$ letters, $\mathrm{SD}=1.4)$ and a lexical frequency greater than 1 per million 11 according to the "Lexique 2" database (New, Pallier, Brysbaert, \& Ferrand, 2004).

Construction of the experimental search displays. During each of the 24 experimental search trial (12 literal search trials and 12 categorical search trials), participants searched for a target word in a sequence of search displays including critical search displays (see Table 1 for examples), plus an additional number of other displays as detailed below. The critical search displays used in each trial included:

- Eighteen "target-related" search displays where the parafoveal word was related to the target word and was either a "related orthographic distractor" (ROD, 9 displays) or a "related semantic distractor" (RSD, 9 displays);

21 - Eighteen "target-unrelated" search displays in which the parafoveal word was unrelated to 22 the target word.

The RODs were words that shared at least their first and last two letters with the target word, while the RSDs were semantic associates of both the target word and its super-ordinate 
1 category (Dampuré et al., 2014). Léger et al. (2012) previously verified the existence of a

2 genuine semantic association between the target word, category name and RSDs.

The target-unrelated search displays were created by taking the target-related search

4 displays from another search trial (see Figure 1A for the following explanation). More 5 precisely, the 24 experimental target words were paired in such a way that the 18 critical 6 target-related search displays used during the search trial for a particular target word (say, 7 "Trial A") were also presented to the same participant during the search trial for another target word (say "Trial B"). Because in Trial B the RODs and RSDs were unrelated to the target word, the target-related displays of Trial A became the 18 target-unrelated displays for

Trial B. In other words, the RODs and RSDs of Trial A became "unrelated orthographic 11 distractors" (UODs, nine displays) or "unrelated semantic distractors" (USDs, nine displays) in Trial B (for an example, see Table 1). Conversely, the target-related search displays of Trial B were inserted as the target-unrelated search displays in Trial A. As a consequence, each search display related to a particular target word was seen twice by each participant: once during the trial where the participant searched for this particular target word, and once as a target-unrelated search display during a trial where the participant searched for another target word. This design allowed us to compare the potentials evoked by the very same search displays in a situation in which the parafoveal words were related to the target word and in a situation in which they were unrelated to the target word (Figure 1A).

\section{INSERT TABLE 1 ABOUT HERE}

The manipulation across the critical search displays of the orthographic or semantic relatedness of the parafoveal words with the target word was associated with a manipulation of the foveal stimulus. Among the nine search displays showing parafoveal words of each of 
1 the four categories defined above (i.e., RODs and RSDs in "target-related" search displays,

2 and UODs or USDs in "target-unrelated" search displays), there were three displays where the

3 foveal stimulus was hash symbols, three displays where the foveal stimulus was a neutral

4 word unrelated to any target word (i.e., an "unrelated neutral distractor" or UND), and three

5 displays where the foveal stimulus was another instance of the same type of distractor word as

6 the parafoveal word, e.g., a parafoveal ROD presented together with a foveal ROD (Table 1

7 and Figure 1A). Hence, each experimental search trial included 12 distinct types of critical

8 search displays; the three instances of each type of display showed the same combination of

9 foveal and parafoveal stimuli, i.e., a parafoveal ROD (alternatively UOD, RSD, or USD)

10 displayed together with foveal hash symbols, with a foveal UND, or with a different foveal

11 ROD (alternatively UOD, RSD, or USD). Across the 12 experimental search trials performed

12 in each search condition (literal or categorical), each participant saw 36 search displays 13 showing the same combination of parafoveal distractors and foveal stimuli once in a situation

14 where the distractors were related to the target word (i.e., RODs or RSDs) and once in a 15 situation where they were unrelated to the target word (i.e., UODs or USDs).

16

Nine parafoveal and three foveal RODs (alternatively UODS, RSDs or USDs) were needed to build the critical search displays associated to each pair of experimental target words. Since only six orthographic and six semantic distractors were available for each target word (Dampuré et al., 2016), each distractor was used twice across the experiment (Table 1).

INSERT FIGURE 1 ABOUT HERE

Additional search displays in experimental search trials. In addition to the 36 critical search displays described above, each experimental search trial included: 
1 - The "target display" where the parafoveal word was the target word, which was always the

2 last display of the search trial.

3 - One to six filler displays that showed one parafoveal and one foveal UND. They were

4 introduced randomly in the sequence of displays in order to vary the length of search trials

5 and reduce the possibility of predicting the time of occurrence of the target.

6 - Five other randomly introduced search displays showing secondary targets (i.e., dollar

7 symbols, $\$ \$ \$ \$ \$$ ) instead of the parafoveal word. The participants were instructed to press

8 the button referring to a "yes" response when seeing these displays. These secondary targets

9 therefore required a response that was different from that required for the distractors ("no")

10 and limited the repetition of the same response and the risk that the participants may come to

11 respond "no" increasingly systematically throughout the trial. Moreover, because these

12 secondary targets could appear at any time, the participants had to sustain their attention, even

13 at the beginning of the search trial (see Dampuré et al., 2016).

14 The target word and secondary targets were presented together with either foveal hash

15 symbols or foveal target-unrelated words in order not to promote a response strategy based on

16 the nature of the foveal stimulus.

17

Filler search trials. As stated above, in addition to the 24 experimental search trials, 22 filler search trials were created in order to have search trials of different lengths and further reduce the possibility of predicting the time of occurrence of the target word. These filler search trials included 4 to 38 search displays and were created around target words that were different from those used in the experimental search trials. Hence, the target word could appear as early as in the fourth search display in some trials. The distractor words used in the filler search trials were all unrelated to the target word. However, the proportions of 
secondary targets and of the different types of foveal stimuli (i.e., hash symbols or words) were the same as in the experimental search trials.

\section{Procedure}

Each participant searched once for each of the 24 experimental target words within the corresponding search trial. The experiment was divided into two sessions of 12 experimental search trials (six pairs of search trials paired together as described above) and 11 filler search trials and lasted for about two hours. One of the sessions took the form of a literal search, with each search trial beginning with the presentation of the exact target word (e.g., search for "corbeau", raven in English). The other session corresponded to a categorical search, in which only the semantic category of the target-word was specified (e.g., search for "un oiseau", $a$ bird in English). The order of presentation of the two sessions was counterbalanced between participants and the 12 experimental search trials presented to a given participant in the literal task were presented in the categorical task to the next participant. The order of presentation of the search trials within a session was randomized, as was the order of the search displays within each search trial, with the exception of the target display, which was always presented last.

In both tasks, each search display was presented one-by-one for 600 milliseconds. Then, masks automatically replaced both the parafoveal word and the foveal stimulus for 1,600 ms (Figure 1). The participants were instructed to fixate the center of the screen but to pay attention only to the word that appeared at the bottom of the screen. For each search display, they had to press a button with the left hand ("no") if they were certain that the word presented parafoveally was not the target word and to press a button with the right hand ("yes") in all other cases. They were instructed to avoid any movement such as blinking 
1 during the presentation of the words and masks. They were asked to blink only when an

2 asterisk appeared at the center of the screen for 2,000 ms after each masking display and to

3 stop when the fixation cross that preceded the next search display appeared for 1,000 ms.

Electroencephalogram (EEG) recording and preprocessing

6

EEGs were recorded via 64 electrodes arranged in the international 10-20 system using the electrode on the left mastoid (M1) as the reference electrode. Eye movements and blinks were recorded using three electrodes, two on the outer canthus of each eye and one in the infra-orbital region of the right eye, thus making it possible to record the electrical activity emitted by the extraocular muscles when the participants made vertical and horizontal eye movements (electrooculogram, EOG). EOG and EEG data were amplified and recorded continuously throughout the experiment using NeuroScan software at a sampling frequency of $500 \mathrm{~Hz}$, with an online $0.1-40 \mathrm{~Hz}$ band-pass filter. Impedances were kept under $5 \mathrm{k} \Omega$. The data were then processed off-line using Brain Analyzer software (Brain Products GmbH, Gilching, Germany). Processing began by applying a 0.1-30 Hz (24 dB/octave) band-pass filter. The plots were re-referenced offline to average mastoids (M1, M2). The data were segmented from -100 to $2,000 \mathrm{~ms}$ relative to the onset of each search display. The $100 \mathrm{~ms}$ of recording time before stimulus presentation was used to calculate the baseline.

\section{Data Analyses}

Because the study was designed to assess the rejection of parafoveal distractor words, the search displays in which either the target-word or a secondary target was presented (i.e., all displays for which participants had to answer "yes") were not included in the analyses. 
1 Similarly, the displays where errors of rejection of parafoveal distractors were made (i.e.,

2 false alarms in which the participant pressed the "yes" button, 9.3\% of the cases) or where the 3 participant did not answer (1.2\% of the cases) were removed from all analyses.

An artifact rejection procedure was then performed to ensure that only the search displays where participants fixated the center of the screen (and not at the parafoveal word 6 located five centimeters below) were included in the analyses. As demonstrated for horizontal eye movements (Stites et al., 2017), Lins, Picton, Berg, and Scherg (1993) showed that there was a linear relationship between the amplitude of vertical eye movements and the size of the corresponding vertical EOG deflection. An eye movement of one degree of visual angle triggers a deflection of about $12 \mu \mathrm{V}$. Since in the present experiment, the parafoveal words 11 were displayed at five degrees of visual angle below the central fixation point, a vertical eye movement toward the parafoveal word would produce a vertical EOG deflection of $60 \mu \mathrm{V}$. To exclude all trials including sizeable vertical eye movements, an automatic artifact inspection algorithm rejecting any electrical activity outside the $-30 \mu \mathrm{V}$ to $30 \mu \mathrm{V}$ range was applied to the vertical EOG traces and resulted in the exclusion of $3.2 \%$ of the search trials (Figure 2). The plots were then subjected to a thorough visual inspection to reject segments containing other artifacts (e.g., noise, muscle activity, eye blinks, etc.), which resulted in the exclusion of $4.1 \%$ of the remaining search trials (Figure 2). Overall, $17.8 \%$ of the search trials were discarded. In the remaining trials, the average amplitude of vertical EOG deflections was very small $(<3 \mu \mathrm{V})$, suggesting that participants did not make any saccade to the parafoveal word.

INSERT FIGURE 2 ABOUT HERE

As stated above, the search displays in which two orthographic or semantic distractors were shown simultaneously in foveal and parafoveal vision were used to check that the 
1 participants did process the foveal stimuli. The search displays in which the orthographic or

2 semantic distractors were displayed only in parafoveal vision were used to compare the

3 impact of orthographic and semantic parafoveal distractors with that of target-unrelated

4 distractors according to the nature of the search task (literal versus categorical) and the nature

5 of the foveal stimulus (hash symbols versus target-unrelated words).

The impact of the presence of parafoveal orthographic or semantic distractors on error rates and log-transformed response time (i.e. the time that elapsed between the appearance of the stimuli and the moment the participant pressed the response button) was analyzed using respectively a logistic ("logit" package) and a linear ("lmer" package) mixed model implemented in "R" software (version 3.4.0). Both models included Task (literal or 11 categorical), Relatedness condition (target-related or target-unrelated distractors), Type of 12 distractor (orthographic or semantic) and Nature of the foveal stimulus (hash symbols, UND, or another instance of the same type of distractor as the parafoveal word) as fixed factors and 14 Participants and Items as random intercepts. Each model also included as many as possible by-item and by-participant random slopes corresponding to the fixed factors (i.e., Dependent 16 variable $\sim$ Task $*$ Relatedness condition * Type of distractor * Nature of the foveal stimulus + $(1+$ Task + Relatedness condition + Type of distractor + Nature of the foveal stimulus 18 Participant $)+(1+$ Task + Relatedness condition $\mid$ Item $))$. The $\chi^{2}$ and probabilities obtained from the deviance table (Type II Wald $\chi^{2}$ tests) are reported for error rates, whereas the $F$ values and probabilities obtained from the analysis of the type III variance table using 21 Satterthwaite approximation for degrees of freedom (Satterthwaite, 1946) are reported for 22 response times. Post-hoc pairwise comparisons were based on the adjusted least-squares means and were performed using the "lsmeans" package in " $\mathrm{R}$ " software. To control for Type

24 I errors, the Hochberg family wise error correction was applied to $p$ values. 
A second set of analyses using linear mixed models focused on the amplitudes of the

2 electroencephalographic potentials evoked by the 12 different types of critical search displays

3 defined above. As expected, the visual examination of the grand averages confirmed the

4 presence of the three following components, which were selected for further analysis.

5 - A frontal positivity from 140 to $220 \mathrm{~ms}$ after word onset, identified as the P2a component.

6 - A frontal negativity between 260 and $320 \mathrm{~ms}$, which corresponds to the N300 component.

7 - A centro-parietal negativity observed between 450 and $550 \mathrm{~ms}$, which corresponds to the $8 \quad$ N400 component.

Following artifact rejection, the single-trial ERP amplitudes of each one of these four components were extracted for each participant in their respective time windows. Six 11 anatomical regions of interest were defined for ERP analyses (Figure 3), i.e., the anterofrontal (EEG electrodes AF3, AF4, AF7, AF8, F7, F8, AFz), frontal (F1 to F6, Fz), frontocentral ( $\mathrm{FC} 1$ to $\mathrm{FC} 6, \mathrm{FCz})$, central $(\mathrm{C} 1$ to $\mathrm{C} 6, \mathrm{Cz})$, centro-parietal $(\mathrm{CP} 1$ to $\mathrm{CP} 6, \mathrm{CPz})$ and parietal (P1 to P6, Pz) regions. Accordingly, the factor Region was included as a fixed factor in the linear mixed models. However, in view of the anatomical distribution of each component on the scalp, the amplitudes of the P2a and N300 components were only analysed over the antero-frontal, frontal, fronto-central and central regions, whereas the amplitude of the N400 component was only analysed over the central, centro-parietal and parietal regions.

The linear mixed model obtained for each ERP component included Task, Relatedness condition, Type of distractor, Nature of the foveal stimulus and Region as fixed factors and Participants and Items as random intercepts. Each model also included as many as possible by-item and by-participant random slopes corresponding to the fixed factors (i.e., Dependent 
$1 \quad$ variable $\sim$ Task $*$ Relatedness condition * Type of distractor * Nature of the foveal stimulus *

2 Region $+(1+$ Task + Relatedness condition + Type of distractor + Nature of the foveal 3 stimulus |Participant $)+(1+$ Task + Relatedness condition |Item)). The $F$ values and

4 probabilities obtained from the analysis of the type III variance table using Satterthwaite 5 approximation for degrees of freedom (Satterthwaite, 1946) are reported. As for the 6 behavioral data, post-hoc pairwise comparisons were based on the adjusted least-square 7 means and were performed applying the Hochberg method for $p$ value adjustment.

\section{Results}

\section{Error Rates}

A preliminary analysis showed that the participants missed more parafoveal target words in the categorical than in the literal task $\left(31.9 \%\right.$ vs. $\left.11.6 \%, \chi^{2}(1)=20.60, p<.001\right)$.

\section{INSERT TABLE 2 ABOUT HERE}

As predicted by hypothesis 1 , the logistic mixed model obtained for error rates (Table 2) revealed a main effect of Task such that participants made more errors in the categorical task than in the literal task $\left(23.7 \%\right.$ vs. $\left.11.3 \%, \chi^{2}(1)=6.68, p<.01\right)$. There was also an

21 interaction between Task and Nature of the foveal stimulus $\left(\chi^{2}(1)=16.16, p<.001\right)$. In accordance with hypothesis 2, post-hoc pairwise comparisons demonstrated that in the literal task, more errors were made when the foveal stimulus was hash symbols rather than a UND $(b=.34, S E=.12, z=2.74, p<.01)$ or than another instance of the same type of distractor as the parafoveal word $(b=.41, S E=.11, z=3.60, p<.001)$. However, Nature of the foveal 
1 stimulus had no significant impact on error rates in the categorical task. Finally, in accordance

2 with hypothesis 3, the model revealed a three-way interaction (Table 2) between Task,

3 Relatedness condition and Type of distractor $\left(\chi^{2}(1)=17.34, p<.001\right)$. The post-hoc pairwise

4 comparisons that were performed using Type of distractor as pivot demonstrated that RODs

5 led to more errors than UODs both in the categorical task $(b=.20, S E=.10, z=1.97, p<.05)$

6 and in the literal task $(b=1.14, S E=.12, z=9.22, p<.001)$ and that the impact of RODs was

7 greater in the latter case. In contrast, RSDs led to more errors than USDs in the categorical 8 task $(b=.48, S E=.11, z=4.50, p<.001)$, but not in the literal task $(b=-.08, S E=.14, z=-$ $9 \quad .58, p=.56)$.

\section{Response times}

As predicted by hypothesis 1 , the linear mixed model obtained for response times (Table 3) revealed a main effect of Task $(F(1,17)=23.30, p<.001)$ with longer response times in the categorical task $(M=935 \mathrm{~ms}, S D=342)$ than in the literal task $(M=685 \mathrm{~ms}, S D$

16 = 274). In accordance with hypothesis 2 , there was also an interaction between Task and Nature of the foveal stimulus $(F(2,605)=9.27, p<.001)$. As for error rates, post-hoc pairwise comparisons demonstrated that in the literal task, response times were shorter when the foveal stimulus was a UND rather than hash symbols $(b=.018, S E=.006, t(130)=3.04$, $p<.01)$ or than another instance of the same type of distractor as the parafoveal word $(b=$ $.014, S E=.004, t(130)=3.09, p<.01)$. However, Nature of the foveal stimulus had no significant impact on error rates in the categorical task. Finally, in accordance with hypothesis 3, the model revealed a three-way interaction (Table 3) between Task, Relatedness condition and Type of distractor $(F(1,12,319)=46.10, p<.001)$. The post-hoc pairwise comparisons 
1 response times than UODs in the literal task $(b=.054, S E=.005, t(130)=11.14, p<.001)$

2 and to marginally slower response times than UODs in the categorical task $(b=.01, S E=$ $3.005, t(157)=1.89, p=.06)$. In contrast, RSDs led to slower response times than USDs in the 4 categorical task $(b=.018, S E=.005, t(147)=3.59, p<.001)$, but not in the literal task $(b=$

\section{INSERT TABLE 3 ABOUT HERE}

\section{ERP results}

Analyses of the P2a component (140 - $200 \mathrm{~ms})$. As predicted by hypotheses 4 and 5, the linear mixed model obtained for the P2a component revealed a four-way interaction between Task, Type of distractor, Relatedness condition and Nature of the foveal stimulus $(F(2,50,633)=8.20, p<.001)$. Follow-up analyses demonstrated that the three-way interaction between Task, Relatedness condition and Nature of the foveal stimulus was significant for both orthographic $(F(2,24,513)=4.28, p<.01)$ and semantic distractors $(F(2$, $26,052)=16.1, p<.001)$. The post-hoc pairwise comparisons that were performed using Nature of the foveal stimulus as pivot revealed that in the literal task, but not in the categorical task (Figure 4), RODs evoked a less positive P2a than UODs when the foveal stimulus was either another $\operatorname{ROD}(b=-.86, S E=.35, t(225)=-2.49, p<.01)$ or hash symbols $(b=-.72, S E=.35, t(237)=-2.04, p<.05)$, but not when the foveal stimulus was a UND $(b=$ $-.01, S E=.35, t(225)=-.03, p=.98)$. In the literal task, RSDs evoked a more positive P2a than USDs (Figure 5) only when the foveal stimulus was hash symbols $(b=1.02, S E=.33$, $t(211)=3.14, p<.01)$. In the categorical task RSDs evoked a more positive P2a than USDs 
1 (Figure 5) only when the foveal stimulus was another $\operatorname{RSD}(b=.85, S E=.34, t(238)=2.51, p$

$2<.01$ ). None of the other post-hoc comparisons was significant (all $p$ values $>.20$ ).

3

4

5

6

7

\section{INSERT FIGURE 4 ABOUT HERE}

Analyses of the $\mathbf{N 3 0 0}$ component $(260$ - $320 \mathrm{~ms})$. Again, the linear mixed model obtained for the N300 component revealed the expected four-way interaction between Task, Type of distractor, Relatedness condition and Nature of the foveal stimulus $(F(2,50,617)=$ 6.93, $p<.001)$. Follow-up analyses demonstrated that the three-way interaction between Task, Relatedness condition and Nature of the foveal stimulus was significant for orthographic distractors $(F(2,24,493)=7.80, p<.001)$, but not for semantic distractors $(F(2$, $26,065)=1.74, p=.18$ ). The post-hoc pairwise comparisons that were performed using Nature of the foveal stimulus as pivot revealed that in the literal task, but not in the categorical task (Figure 4), RODs evoked a more negative N300 than UODs when the foveal stimulus was another ROD $(b=-.99, S E=.35, t(205)=-2.84, p<.01)$. None of the other post-hoc comparisons was significant (all $p$ values $>.20$ ).

\section{INSERT FIGURE 5 ABOUT HERE}

\section{Analyses of the $\mathbf{N 4 0 0}$ component $(450$ - $550 \mathrm{~ms})$. The linear mixed model obtained} for the N400 component revealed a significant main effect of Nature of the foveal stimulus $(F(2,26)=30.32, p<.001)$. Post-hoc pairwise comparisons demonstrated (Figure 6) that the critical search displays evoked a more negative N400 when the foveal stimulus was hash symbols rather than a UND $(b=-1.88, S E=.25, t(21)=7.64, p<.001)$ or than another instance of the same type of distractor as the parafoveal word $(b=-1.59, S E=.23, t(23)=$ 
$16.96, p<.001)$. In addition, the parafoveal distractors evoked a marginally more negative

2 N400 when the foveal stimulus was another instance of the same type of distractor as the 3 parafoveal word rather than a UND $(b=.29, S E=.16, t(33)=1.84, p=.07)$. There was also a 4 marginal main effect of Task $(F(1,22)=3.66, p=.07)$, such that the critical search displays 5 evoked a more negative N400 in the literal task than in the categorical task (Figure 6).

6

\section{INSERT FIGURE 6 ABOUT HERE}

\section{Discussion}

Overall impact of the foveal and task-related working memory loads on parafoveal processing

As expected, the manipulation of the task-related working memory load had a strong impact on the rejection of parafoveal distractor words. Indeed, whatever the foveal stimulus, the categorical task was associated with much slower response times and a larger number of errors of rejection of parafoveal distractors than the literal task. Moreover, the participants missed more parafoveal target words in the categorical than in the literal task. The ERP data revealed that all parafoveal distractors tended to evoke a more negative $\mathrm{N} 400$ in the literal task than in the categorical task, which was not the case when they were presented at the participants' fovea (Dampuré et al., 2016). Since the amplitude of the N400 component would reflect the depth of word processing (Chwilla, Brown, \& Hagoort, 1995), this suggests that the parafoveal distractors were processed more deeply in the literal task than in the categorical task. Altogether, the results show that in the categorical task, fewer resources would be available for the processing of words in parafoveal vision. The distractor rejection process 
1 would be slower and less accurate because more cognitive resources are needed for the

2 maintenance of the target template in memory.

In the search displays in which only parafoveal distractors were shown, the

4 manipulation of the nature of the foveal stimulus (i.e., hash symbols versus UND) had an

5 impact on both the duration and accuracy of parafoveal distractor rejection in the literal task,

6 but not in the categorical task. This result suggests that in the categorical task, the task-related

7 working memory load was already maximal, which prevented the manipulation of the foveal

8 load from having a significant impact on error rates and response times.

In the literal task, unexpectedly, response times were actually longer and error rates

lower when the foveal stimulus was a UND rather than hash symbols. At first sight, this

11 suggests that parafoveal processing was more efficient when the foveal stimulus was a UND,

12 i.e., in a situation where the foveal load should be higher than with foveal hash symbols and thus in contradiction with Lavie's Load Theory (De Fockert, 2013; Lavie \& De Fockert, 2005). However, a closer look at the data revealed that in the search displays where the nature of the foveal stimulus was manipulated, most of the errors of distractor rejection observed in the literal task were actually made on parafoveal related orthographic distractors (RODs, see Table 2). Consequently, most of the increase in error rates observed when the foveal stimulus was hash symbols rather than a UND was due to a higher number of errors on RODs, i.e., on words that were visually similar to the target word. Similarly, the increase in the time needed to reject parafoveal distractors observed when the foveal stimulus was hash symbols (see

21 Table 3) was mainly due to a $52 \mathrm{~ms}$ average increase in the rejection time of parafoveal 22 RODs. In contrast, the rejection times of the other types of distractors increased by only $4 \mathrm{~ms}$ (RSDs) and $22 \mathrm{~ms}$ (USDs) or even decreased by $3 \mathrm{~ms}$ (UODs). This pattern of results may explain the apparent discrepancy between the data and the hypotheses that were made regarding the impact of foveal load on parafoveal processing. 
1 Indeed, according to Dampuré et al. (2016), the identification and rejection of distractor

2 words during visual search tasks involve two separate mechanisms. First, the features of 3 distractor words are used to quickly detect similarities with the target template stored in 4 working memory, and then the distractors that have been preselected based on their visual or

semantic similarity with the template are selected for further analysis and ultimately rejected.

In the context of the present experiment, the lower foveal load associated with hash symbols would allow participants to more easily detect the visual similarities between the parafoveal distractors and the target compared with when the foveal stimulus is a UND. As stated above, participants had to tell whether they were "certain that the word presented parafoveally was not the target word". When the foveal stimulus is a UND, participants may be less prone to detect the visual similarity between the target template and RODs, and may thus be more certain that RODs are not the target and must be rejected. In other words, in the high foveal load condition, a higher proportion of RODs would not be preselected for further analysis, despite their visual similarity with the target. Conversely, when the foveal stimulus is hash symbols and the foveal load lower, more cognitive resources may be available to detect the visual similarities between RODs and the target, and more RODs would be preselected for further analysis; this would lead to slower rejection times and more errors of rejection of RODs than when the foveal stimulus is a UND.

This interpretation is supported by the ERP data, which show that whatever the search task, parafoveal distractors evoked a more negative N400 when the foveal stimulus was hash symbols rather than a UND or than another instance of the same type of distractor as the parafoveal word. The modulation of the N400 by the nature of the foveal stimulus suggests that in contrast with the meaningless hash symbols, the words presented on the fovea (i.e., a UND or the same type of distractor as the parafoveal word) were semantically processed (e.g., 
1 Kutas \& Federmeier, 2011). Hence, these data confirm that more resources were available to

2 process the parafoveal distractors when the foveal stimulus was hash symbols. ${ }^{1}$

If this interpretation is correct, the lower foveal load associated with hash symbols

4 should allow participants to more easily detect the visual and semantic similarities between

5 the parafoveal distractors and the target. Consequently, the ERPs evoked by parafoveal

6 distractors in the literal task should be more modulated by their orthographic or semantic

7 relatedness to the target when the foveal stimulus is hash symbols rather than a UND. The

8 ERP data reported below confirm this interpretation.

Impact of cognitive load on the detection of visual and semantic similarities between the

parafoveal distractors and the target

The respective impacts of the working memory load and foveal cognitive load on the detection of visual and semantic similarities between the parafoveal distractors and the target were tested by observing the ERP components evoked by parafoveal distractors in the search displays in which only parafoveal distractors were present. significantly different from those evoked by their respective controls (i.e., parafoveal UODs and USDs), but only when hash symbols rather than a UND were displayed at the center of the screen. This confirms that, as hypothesized in the previous paragraph, the higher error rates and slower response times obtained when hash symbols were displayed at the center of

\footnotetext{
${ }^{1}$ Close examination of the ERP data revealed that a centro-parietal positivity observed between 310 and $380 \mathrm{~ms}$ and identified as the P300 component (see Figure 6) was strongly modulated by the nature of the foveal stimulus $(\mathrm{F}(2,36)=27.99, \mathrm{p}<.001)$. The critical search displays evoked a more positive P300 when the foveal stimulus was hash symbols rather than a UND $(b=1.57, \mathrm{SE}=.21, \mathrm{t}(25)=7.42, \mathrm{p}<.001)$ or than another instance of the same type of distractor as the parafoveal word $(b=1.53, \mathrm{SE}=.23, \mathrm{t}(24)=6.72, \mathrm{p}<.001)$. Since several authors reported that the amplitude of the P300 component would index the amount of cognitive resources available for monitoring events in the visual periphery (Kramer, Sirevaag, \& Hughes, 1988; Polich, 2007), this results confirms that more resources were available for parafoveal processing when the foveal stimulus was hash symbols.
} 
1 the screen were probably due to a better detection of visual similarities between parafoveally

2 presented words and the target template.

3

Parafoveal orthographic distractors. When the foveal stimulus was hash symbols, parafoveal RODs evoked a less positive P2a than their controls (UODs) in the literal task. Moreover, more errors and longer response times were obtained with RODs than with UODs. In contrast, parafoveal RODs had no impact on the P2a component in the categorical task. In accordance with the hypothesis that the impact of RODs would increase when the task-related working memory load decreases, their impact on error rates and response times was higher in the literal task than in the categorical task.

In the literal task, the impact of parafoveal RODs on the P2a component, which would reflect the early detection of similarities with the target template, was actually similar to that of the foveal RODs described by Dampuré et al. (2016). Thus, when both the foveal and taskrelated working memory loads were low, participants were able to detect the similarities between parafoveal distractors and the target template as well as if the distractors had been presented on the participants' fovea. As discussed more extensively in Dampuré et al., the decrease in P2a amplitude observed in the literal task would reflect the detection of a visual similarity between RODs and target words at a sublexical level (Dambacher, Kliegl, Hofmann, \& Jacobs, 2006; Lei, Li, Long, Li, Chen, et al., 2010).

In contrast with foveal RODs (see Dampuré et al., 2016), parafoveal RODs did not evoke a more positive P2a than their controls in the categorical task. The absence of modulation of these components by parafoveal RODs in the categorical task suggests that when the task-related working memory load increased, participants failed to detect any similarity between RODs and the target and confirms that in the categorical task, fewer cognitive resources would be available for the processing of parafoveal words. 
Parafoveal semantic distractors. When hash symbols were displayed at the center of the screen, RSDs evoked a more positive P2a than their controls in the literal task only.

4 Compared with USDs, the presence of RSDs was associated with increased error rates in the categorical task but not in the literal task, but the response times were not significantly modified. In accordance with previous visual search studies, which reported that semantic distractors had a weaker impact on attention guidance than orthographic distractors (Dampuré et al., 2014; Léger et al., 2012), parafoveal semantic distractors had a weaker impact on behavioral responses than parafoveal orthographic distractors. The increase in error rates observed in the categorical task is in line with the high proportion of errors made by 11 participants in real categorical visual search tasks (Dampuré et al., 2014; Léger et al., 2012), where semantic distractors are quite often erroneously selected as the target word. Interestingly, whereas in the present study parafoveal RSDs evoked a more positive P2a than their controls in the literal task only, Dampuré et al. (2016) reported that foveal RSDs evoked a more positive $\mathrm{P} 2 \mathrm{a}$ than their controls in the categorical task only. As discussed by Dampuré et al, an increased P2a amplitude would reflect the early detection of a similarity with the target word at a lexico-semantic level rather than at a visual, sublexical level (Baccino \& Manunta, 2005; Barber et al., 2013; Blanchet, Gagnon, \& Bastien, 2007; Federmeier, Mai, \& Kutas, 2005; Lee, Liu, \& Tsai, 2012). Hence, this confirms again that the task-related cognitive load is too high in the categorical task to allow efficient parafoveal 21 processing. In the literal task, in contrast, more cognitive resources would be available for the 22 early detection of target-distractor semantic similarity. However, the question arises as to why 23 Dampuré et al. did not find any significant impact of foveal RSDs on the P2a component in 24 the literal task. One explanation may be that when the distractors are presented on the fovea, 25 semantic distractors are not preselected because they do not share any visual feature with the 
1 target word. Parafoveal RSDs, in contrast, may need further processing to be rejected based

2 on their visual dissimilarity with the target word.

Unlike the foveal RSDs (Dampuré et al., 2016), the parafoveal RSDs did not evoke a

4 less negative N300 than the parafoveal USDs in either the literal task or the categorical task.

5 As discussed by Dampuré et al., the decrease in N300 amplitude observed with foveal RSDs

6 would reflect easier access to word meaning (Cristescu \& Nobre, 2008). The meaning of

7 foveal RSDs should be more easily accessed than that of USDs during the search task because

8 the former share semantic features with the target template. The absence of modulation of the

9 N300 component by parafoveal RSDs suggests that participants failed to detect the semantic

link between RSDs and the target and confirms that whatever the search task, fewer cognitive

11 resources would be available for parafoveal word processing than for foveal word processing.

12

Potentials evoked by the search displays showing both foveal and parafoveal distractors.

As expected, when the word displayed at the center of the screen duplicated the word that was presented parafoveally, the modulations of the P2a and N300 components by orthographic and semantic distractors compared to unrelated distractors became more similar to those obtained by Dampuré et al. (2016) with just the foveal distractors and no word in parafoveal vision. Indeed, in the literal task, the displays showing two RODs evoked a less positive P2a and more negative N300 than those showing two UODs as did foveal RODs compared to foveal UODs in Dampuré et al.'s study, whereas when presented with foveal hash symbols, parafoveal RODs only had an impact on the P2a component compared to parafoveal UODs. Similarly, the displays showing two RSDs evoked a more positive P2a than those showing two USDs in the categorical task as did foveal RSDs compared to foveal USDs in Dampuré et al.'s study, whereas when presented with foveal hash symbols, parafoveal 
1 RSDs evoked a more positive P2a than parafoveal USDs in the literal task. Consequently, the

2 data obtained with the displays that included two RODs or two RSDs suggest that despite the

3 instructions to attend only to the parafoveal stimuli, the participants did process the stimulus

4 appearing at the center of the screen.

6 Parafoveal and foveal mechanisms of visual search for words

7

Visual search models conceive of the search activity as interplay between two main attentional mechanisms (Deco \& Zihl, 2006; Desimone \& Duncan, 1995; Wolfe, 2007; Zelinsky, 2008). Attentional guidance mechanisms determine the order in which the different objects present in the visual field are examined according to the number of features they share with the target. Then, once an object is fixated, a recognition mechanism categorizes it as the target or a distractor.

In the modified visual search tasks for words used here and by Dampuré et al. (2016), the modulations of the P2a component by orthographic and/or semantic distractors would reflect attentional guidance mechanisms. Fast pre-attentive mechanisms occurring within 200 ms after the presentation of words would select the best target candidates based on their visual similarity and/or semantic relatedness with the target template. Indeed, the timing of the P2a component is short enough to make it a relevant marker in the understanding of eye movement control (Reichle, Pollatsek, \& Rayner, 2012). The present study complements this picture by showing that the early word selection mechanism that underlies attention guidance and is reflected by modulations of the P2a component can be deployed simultaneously over both the foveal and the parafoveal visual fields. In both parts of the visual field, this mechanism is based on comparisons between the features of the words present in the display and those of the target stored and activated in memory. In addition, the data demonstrate that 
1 the efficiency of the detection of similarities between parafoveal distractor words and the

2 target depend on the amount of cognitive resources that are available to process parafoveal

3 stimuli. This extends previous visual search data that demonstrated the sensitivity of

4 attentional guidance mechanisms to cognitive load (Soto et al., 2008; Zhang et al., 2011) to

5 the case of words.

More precisely, in accordance with initial hypotheses, the efficiency of attentional 7 guidance mechanisms decreased when the working memory load increased. Indeed, parafoveal distractors were rejected faster and more accurately in the literal task than in the categorical task. In the literal task, the efficiency of attention guidance by parafoveal distractors was also modulated by the amount of cognitive resources used to process the

11 foveal stimuli, i.e., the foveal load. The potentials evoked by parafoveal distractors were impacted by their visual similarity or semantic relatedness with the target only when hash symbols rather than words were displayed at the center of the screen.

In contrast with attentional guidance mechanisms, the distractor rejection mechanisms that follow the preselection of distractors for further examination would not reach full efficiency and awareness as long as the distractors are not directly fixated. Indeed, as detailed above, the parafoveal distractor words did not have any impact on the N300 and N400 components, which confirms that they were not processed as deeply as when they were presented alone at the participants' fovea (Dampuré et al., 2016). Conversely, the later N300 and N400 components would be more related to the distractor rejection mechanisms taking 21 place once a distractor word is fixated.

Altogether, present and past studies (Dampuré et al., 2016) point towards a unified conception of the attentional mechanisms used during visual search for visual stimuli (e.g., objects, words, or pictures). The early attention guidance mechanisms, which are deployed over the whole visual field to detect visual and semantic similarities between what is 
1 perceived and the target template, appear to be very sensitive to cognitive load. Despite the

2 fact that these processes do not reach awareness, their efficiency would decrease as the task3 related working memory load and/or the foveal load increase. In contrast, the target

4 recognition mechanisms would only reach full efficiency and awareness in foveal vision, in

5 accordance with previous models of visual search (e.g., Zelinsky, 2008) that claim that in 6 natural, speeded search situations, foveal inspection is needed for full, conscious 7 identification of the visual stimuli.

\section{$9 \quad$ Limitations and research perspectives}

The present data are consistent with most of the gaze number and average gaze duration data obtained in previous eyetracking studies of the impact of orthographic and semantic distractors during actual visual search for words (Dampuré et al., 2014; Léger et al., 2012). However, some of the effects found in actual visual search do not match the present data. In Dampuré et al. (2014), orthographic distractors attracted participants' gaze in the literal task regardless of the type of words they were currently looking at, which suggests that parafoveal orthographic distractors should have a significant impact on the potentials evoked by parafoveal words regardless of the foveal stimulus. Nevertheless, parafoveal orthographic distractors only had an impact on ERP components when hash symbols were displayed at the center of the screen. One possible explanation is that during active search tasks, visual

21 information can be grabbed from various words at each glance, and information about the 22 whole display can accumulate over time (Wolfe, 1994) to be used later in the task. In the present experiment, in contrast, parafoveal processing of distractor words could only be

24 performed once. Another possible explanation may be that in the present experiment, 25 participants engaged in less parafoveal processing of the distractors than when a real saccade 
1 is triggered toward them (Deubel \& Schneider, 1996), even though similar modulations of the

2 P2a amplitude by parafoveal words were found in active reading-like task (Baccino \&

3 Manunta, 2005). This discrepancy between the actual visual search data and the present

4 results questions the transferability of the present data to the situations where multiple words

5 are presented simultaneously in peripheral vision. In other words, how verbal information

6 accumulates over time without awareness and how this impacts eye movements in real visual

7 search tasks must be further investigated.

Interestingly, the data are also shedding light on observations made in the context of reading activities. Obviously, visual search for words is quite different from usual reading tasks, in particular because the search task consists mostly in rejecting as quickly as possible

11 the words that are not the target, whereas reading tasks aim at integrating word meanings into 12 an understandable whole. However, recent work suggests that during reading, the amount of processing of parafoveally-previewed words also depends on the current foveal load and available attentional resources (Payne et al., 2016; Rayner et al., 2014; Schotter, 2013). In the present study, lexico-semantic processing of parafoveal words seemed to occur even earlier 16 (i.e., during the first $200 \mathrm{~ms}$ ) than in reading studies (Barber et al., 2013; Federmeier et al., 17 2005; Cristescu \& Nobre, 2008). Another difference between the reading literature and the present study is that most of the studies that investigated parafoveal word processing in reading manipulated the foveal load by controlling for the lexical frequency of the foveal word. High frequency words

21 decrease the foveal load and increase parafoveal word processing compared to low frequency words (Henderson \& Ferreira, 1990; Hohenstein \& Kliegl, 2014; Schroyens et al., 1999). In the present study, as in a few reading studies (e.g. Schroyens et al., 1999), the foveal load was manipulated by modifying the relevance of the foveal stimulus for the current task. According to Lavie's Load Theory (Lavie \& Robertson, 2001), task-relevant stimuli (i.e., words in the 
1 present study) would increase the foveal load compared to task-irrelevant stimuli (i.e., hash

2 symbols or z-strings in Schroyens et al.). Altogether, future studies on the impact of foveal

3 load on parafoveal word processing should take into account the many parameters that may

4 influence foveal load, i.e., the processing difficulty and task-relevance of the foveal stimulus,

5 but also the task-related time constraints. A better knowledge of the word processing

6 principles common to all verbal tasks should pave the way for the elaboration of a unified

7 model of visual word processing (Reichle et al., 2012).

8

9

10

11

12

\section{Acknowledgments}

Julien Dampuré was supported by a Ph.D. fellowship from the Direction Générale de l'Armement (DGA, French Ministry of Defense) under the supervision of Didier Bazalgette. The authors would like to thank Jean-François Rouet for his helpful comments and advice during the preparation of this experiment, and all of the participants for their time. The experiments were performed using the human behavior analysis facilities of the Maison des Sciences de l'Homme et de la Société (MSHS) de Poitiers (USR 3565 CNRS - Université de Poitiers).

\section{References}

Baccino, T., \& Manunta, Y. (2005). Eye-fixation-related potentials: Insight into parafoveal processing. Journal of Psychophysiology, 19(3), 204-215.

Barber, H. A., Ben-Zvi, S., Bentin, S., \& Kutas, M. (2011). Parafoveal perception during sentence reading? An ERP paradigm using rapid serial visual presentation (RSVP) with flankers. Psychophysiology, 48(4), 523-531.

Barber, H. A., Doñamayor, N., Kutas, M., \& Münte, T. (2010). Parafoveal N400 effect during sentence reading. Neuroscience Letters, 479(2), 152-156. 
Barber, H. A., Van der Meij, M., \& Kutas, M. (2013). An electrophysiological analysis of contextual and temporal constraints on parafoveal word processing. Psychophysiology, 50(1), 48-59.

Becker, S. I. (2011). Determinants of dwell time in visual search: similarity or perceptual difficulty? PloS One, 6(3), e17740.

Belke, E., Humphreys, G. W., Watson, D. G., Meyer, A. S., \& Telling, A. L. (2008). Topdown effects of semantic knowledge in visual search are modulated by cognitive but not perceptual load. Perception \& Psychophysics, 70(8), 1444-1458.

Blanchet, S., Gagnon, G., \& Bastien, C. (2007). Event-related potential study of dynamic neural mechanisms of semantic organizational strategies in verbal learning. Brain Research, 1170, 59-70.

Boot, I., \& Pecher, D. (2008). Word recognition is affected by the meaning of orthographic neighbours: Evidence from semantic decision tasks. Language and Cognitive Processes, 23(3), 375-393.

Calvo, M. G., \& Castillo, M. D. (2009). Semantic word priming in the absence of eye fixations: Relative contribution of overt and covert attention. Psychonomic Bulletin and Review, 16, 51-56.

Chwilla, D. J., Brown, C. M., \& Hagoort, P. (1995). The N400 as a function of the level of processing. Psychophysiology, 32(3), 274-285.

Coulson, S., Federmeier, K. D., Van Petten, C., \& Kutas, M. (2005). Right hemisphere sensitivity to word-and sentence-level context: evidence from event-related brain potentials. Journal of Experimental Psychology: Learning, Memory, and Cognition, 31(1), 129-147.

Cristescu, T. C., \& Nobre, A. C. (2008). Differential modulation of word recognition by semantic and spatial orienting of attention. Journal of Cognitive Neuroscience, 20(5), 787-801.

Dambacher, M., Kliegl, R., Hofmann, M., \& Jacobs, A. M. (2006). Frequency and predictability effects on event-related potentials during reading. Brain Research, 1084(1), 89-103.

Dampuré, J., Benraiss, A., \& Vibert, N. (2016). Task-dependent modulation of word processing mechanisms during modified visual search tasks. The Quarterly Journal of Experimental Psychology, 69(6), 1145-1163. 
1 Dampuré, J., Ros, C., Rouet, J.-F., \& Vibert, N. (2014).Task-dependent sensitization of perceptual and semantic processing during visual search for words. Journal of Cognitive Psychology, 26 (5), 530-549.

De Fockert, J. W. (2013). Beyond perceptual load and dilution: a review of the role of working memory in selective attention. Frontiers in Psychology, 4, 287.

Deco, G., \& Zihl, J. (2006). The neurodynamics of visual search. Visual Cognition, 14(4-8), 1006-1024.

Desimone, R., \& Duncan, J. (1995). Neural mechanisms of selective visual attention. Annual Review of Neuroscience, 18, 193-222.

Deubel, H., \& Schneider, W. X. (1996). Saccade target selection and object recognition: Evidence for a common attentional mechanism. Vision Research, 36, 1827-1837.

Dimigen, O., Kliegl, R., \& Sommer, W. (2012). Trans-saccadic parafoveal preview benefits in fluent reading: A study with fixation-related brain potentials. Neuroimage, 62(1), 381-393.

Downing, P. E. (2000). Interactions between visual working memory and selective attention. Psychological Science, 11(6), 467-473.

Duncan, J., \& Humphreys, G. W. (1989). Visual search and stimulus similarity. Psychological Review, 96, 433-458.

Federmeier, K. D., Mai, H., \& Kutas, M. (2005). Both sides get the point: Hemispheric sensitivities to sentential constraint. Memory \& Cognition, 33(5), 871-886.

Franklin, M. S., Dien, J., Neely, J. H., Huber, E., \& Waterson, L. D. (2007). Semantic priming modulates the N400, N300, and N400RP. Clinical Neurophysiology, 118(5), 10531068 .

Grainger, J. \& Jacobs, A. M. (1996). Orthographic processing in visual word recognition: A multiple read-out model. Psychological Review, 103, 518-565.

Harris, C. R., \& Pashler, H. (2004). Attention and the processing of emotional words and names - Not so special after all. Psychological Science, 15(3), 171-178.

Heil, M., Rolke, B., \& Pecchinenda, A. (2004). Automatic semantic activation is no myth. Psychological Science, 15(12), 852-857.

Henderson, J.M., \& Ferreira, F. (1990). Effects of foveal processing difficulty on the perceptual span in reading: Implications for attention and eye movement control. Journal of Experimental Psychology: Learning, Memory, and Cognition, 16(3), 417429. 
1 Hohenstein, S., \& Kliegl, R. (2014). Semantic preview benefit during reading. Journal of Experimental Psychology: Learning, Memory, and Cognition, 40(1), 166-190.

Holmes, D. L., Cohen, K. M., Haith, M. M., \& Morrison, F. J. (1977). Peripheral visual processing. Perception \& Psychophysics, 22(6), 571-577.

Hout, M. C., \& Goldinger, S. D. (2015). Target templates: The precision of mental representations affects attentional guidance and decision-making in visual search. Attention, Perception, \& Psychophysics, 77(1), 128-149.

Huettig, F., \& McQueen, J. M. (2007). The tug of war between phonological, semantic and shape information in language-mediated visual search. Journal of Memory and Language, 57(4), 460-482.

Huettig, F., Olivers, C. N. L., \& Hartsuiker, R. J. (2011). Looking, language and memory: Bridging research from the visual world and visual search paradigms. Acta Psychologica, 137, 138-150.

Jacob, M., \& Hochstein, S. (2009). Comparing eye movements to detected versus undetected target stimuli in an Identity Search task. Journal of Vision, 9(5):20, 1-16.

Kornrumpf, B., \& Sommer, W. (2015). Modulation of the attentional span by foveal and parafoveal task load: An ERP study using attentional probes. Psychophysiology, 52(9), 1218-1227.

Kramer, A. F., Sirevaag, E. J., \& Hughes, P. R. (1988). Effects of foveal task load on visual-spatial attention: event-related brain potentials and performance. Psychophysiology, 25(5), 512-531.

Kutas, M., \& Federmeier, K. D. (2011). Thirty years and counting: finding meaning in the N400 component of the Event-Related Brain Potential (ERP). Annual Review of Psychology, 62, 621-647.

Lavie, N., \& De Fockert, J. (2005). The role of working memory in attentional capture. Psychonomic Bulletin \& Review, 12(4), 669-674.

Lavie, N., \& Robertson, I. H. (2001). The role of perceptual load in neglect: rejection of ipsilesional distractors is facilitated with higher central load. Journal of Cognitive Neuroscience, 13(7), 867-876.

Lavie, N., Hirst, A., De Fockert, J. W., \& Viding, E. (2004). Load theory of selective attention and cognitive control. Journal of Experimental Psychology: General, 133(3), 339.

Lee, C. Y., Liu, Y. N., \& Tsai, J. L. (2012). The time course of contextual effects on visual word recognition. Frontiers in Psychology, 3, 1-13. 
Léger, L., \& Tijus, C. (2007). L'effet de l'hétérogénéité sémantique dans la détection de mots. Psychologie Francaise, 52(3), 367-385.

Léger, L., Rouet, J.-F., Ros, C., \& Vibert, N. (2012). Orthographic versus semantic matching in visual search for words within lists. Canadian Journal of Experimental Psychology, 66, 32-43.

Lei, Y., Li, F. H., Long, C. Q., Li, P., Chen, Q. F., Ni, Y. Y., \& Li, H. (2010). How does typicality of category members affect the deductive reasoning? An ERP study. Experimental Brain Research, 204(1), 47-56.

Li, N., Niefind, F., Wang, S., Sommer, W., \& Dimigen, O. (2015). Parafoveal processing in reading Chinese sentences: Evidence from event- related brain potentials. Psychophysiology, 52(10), 1361-1374.

Lins, O. G., Picton, T. W., Berg, P., \& Scherg, M. (1993). Ocular artifacts in recording EEGs and event-related potentials. II: Source dipoles and source components. Brain Topography, 6, 65-78.

López-Peréz, P. J., Dampuré, J., Hernández-Cabrera, J. A., \& Barber, H. A. (2016). Semantic parafoveal-on-foveal effects and preview benefits in reading: Evidence from Fixation Related Potentials. Brain and Language, 162, 29-34.

Luck, S. J., \& Hillyard, S. A. (1994). Electrophysiological correlates of feature analysis during visual search. Psychophysiology, 31(3), 291-308.

Meyer, A. S., Belke, E., Telling, A. L., \& Humphreys, G. W. (2007). Early activation of object names in visual search. Psychonomic Bulletin \& Review, 14(4), 710-716.

Moores, E., Laiti, L., \& Chelazzi, L. (2003). Associative knowledge controls deployment of visual selective attention. Nature Neuroscience, 6(2), 182-189.

Neely, J. H. (1977). Semantic priming and retrieval from lexical memory: Roles of inhibitionless spreading activation and limited-capacity attention. Journal of Experimental Psychology: General, 106(3), 226-254.

New, B., Pallier, C., Brysbaert, M., \& Ferrand, L. (2004). Lexique 2: A new French lexical database. Behavior Research Methods Instruments \& Computers, 36, 516-524.

Olivers, C. N. L., Meijer, F., \& Theeuwes, J. (2006). Feature-based memory-driven attentional capture: visual working memory content affects visual attention. Journal of Experimental Psychology: Human Perception and Performance, 32(5), 12431265. 
Payne, B. R., Stites, M. C., \& Federmeier, K. D. (2016). Out of the corner of my eye: Foveal semantic load modulates parafoveal processing in reading. Journal of Experimental Psychology: Human Perception and Performance, 42(11), 1839-1857

Pomplun, M., Reingold, E. M., \& Shen, J. (2001). Investigating the visual span in comparative search: The effects of task difficulty and divided attention. Cognition, 81(2), B57-B67.

Potts, G. F., \& Tucker, D. M. (2001). Frontal evaluation and posterior representation in target detection. Cognitive Brain Research, 11(1), 147-156.

Potts, G. F., Liotti, M., Tucker, D. M., \& Posner, M. I. (1996). Frontal and inferior temporal cortical activity in visual target detection: Evidence from high spatially sampled event-related potentials. Brain Topography, 9(1), 3-14.

Potts, G. F., Patel, S. H., \& Azzam, P. N. (2004). Impact of instructed relevance on the visual ERP. International Journal of Psychophysiology, 52(2), 197-209.

Rayner, K. (1998). Eye movements in reading and information processing: 20 years of research. Psychological Bulletin, 124, 372-422.

Rayner, K. (2009). Eye movements and attention in reading, scene perception, and visual search. The Quarterly Journal of Experimental Psychology, 62(8), 1457-1506.

Rayner, K., Schotter, E. R., \& Drieghe, D. (2014). Lack of semantic parafoveal preview benefit in reading revisited. Psychonomic Bulletin \& Review, 21(4), 1067-1072.

Reichle, E. D., Pollatsek, A., \& Rayner, K. (2012). Using EZ Reader to simulate eye movements in nonreading tasks: A unified framework for understanding the eyemind link. Psychological Review, 119(1), 155.

Rodd, J. M. (2004). When do leotards get their spots? Semantic activation of lexical neighbors in visual word recognition. Psychonomic Bulletin \& Review, 11, 434-439.

Ruz, M., Madrid, E., Lupiañez, J., \& Tudela, P. (2003). High density ERP indices of conscious and unconscious semantic priming. Cognitive Brain Research, 17, 719731.

Satterthwaite, F. E. (1946). An approximate distribution of estimates of variance components. Biometrics Bulletin, 2(6), 110-114.

Schmidt, J., MacNamara, A., Proudfit, G. H., \& Zelinsky, G. J. (2014). More target features in visual working memory leads to poorer search guidance: Evidence from contralateral delay activity. Journal of vision, 14(3), 8-8.

Schotter, E. R. (2013). Synonyms provide semantic preview benefit in English. Journal of Memory and Language, 69(4), 619-633. 
Schroyens, W., Vitu, F., Brysbaert, M., \& d'Ydewalle, G. (1999). Eye movement control during reading: Foveal load and parafoveal processing. The Quarterly Journal of Experimental Psychology: Section A, 52(4), 1021-1046.

Soto, D., \& Humphreys, G. W. (2007). Automatic guidance of visual attention from verbal working memory. Journal of Experimental Psychology: Human Perception and Performance, 33(3), 730-737.

Soto, D., Heinke, D., Humphreys, G. W., \& Blanco, M. J. (2005). Early, involuntary topdown guidance of attention from working memory. Journal of Experimental Psychology: Human Perception and Performance, 31, 248-261.

Stites, M. C., Payne, B. R., \& Federmeier, K. D. (2017). Getting ahead of yourself: Parafoveal word expectancy modulates the N400 during sentence reading. Cognitive, Affective, \& Behavioral Neuroscience, 17(3), 475-490.

Telling, A. L., Kumar, S., Meyer, A. S., \& Humphreys, G. W. (2010). Electrophysiological evidence of semantic interference in visual search. Journal of Cognitive Neuroscience, 22(10), 2212-2225.

Veldre, A., \& Andrews, S. (2015). Parafoveal preview benefit is modulated by the precision of skilled readers' lexical representations. Journal of Experimental Psychology: Human Perception and Performance, 41(1), 219.

Williams, L. J. (1988). Tunnel vision or general interference? Cognitive load and attentional bias are both important. The American Journal of Psychology, 101(2), 171-191.

Wolfe, J. M. (1994). Guided search 2.0 - A revised model of visual search. Psychonomic Bulletin \& Review, 1(2), 202-238.

Wolfe, J. M. (2007). Guided Search 4.0: Current Progress with a model of visual search. In W. Gray (Ed.), Integrated Models of Cognitive Systems (pp. 99-119). New York: Oxford University Press.

Wolfe, J. M., Butcher, S. J., Lee, C., \& Hyle, M. (2003). Changing your mind: on the contributions of top-down and bottom-up guidance in visual search for feature singletons. Journal of Experimental Psychology: Human Perception and Performance, 29(2), 483-502.

Wolfe, J. M., Horowitz, T. S., Kenner, N., Hyle, M., \& Vasan, N. (2004). How fast can you change your mind? The speed of top-down guidance in visual search. Vision Research, 44(12), 1411-1426.

Yan, M., Richter, E. M., Shu, H., \& Kliegl, R. (2009). Readers of Chinese extract semantic information from parafoveal words. Psychonomic bulletin \& review, 16(3), 561-566. 
1 Zhang, W., Li, N., Wang, X., \& Wang, S. (2015). Integration of Sentence-Level Semantic topographical variables used for the study of the ERP components.

\section{Legends of the figures} search display was presented and the instructions given to the participant. (i.e. a saccade) and a blink.

Figure 1. Experimental design. The first panel (A) shows the different types of search displays that were presented in two paired, randomly presented search trials (i.e., Trial A and Trial B) with the status of the displayed words (i.e., target, $\mathrm{R}^{*} \mathrm{D}$ for either ROD or RSD, $\mathrm{U}^{*} \mathrm{D}$ for either UOD or USD, or UND) as a function of the trial in which they were presented (see Table 1 for an example). Note that the 18 critical search displays which show parafoveal distractors (ROD or RSD) related to one of the two target words (say, target A) are presented another time to the same participant during trial $\mathrm{B}$, where they are not related to the target word and thus become UOD and USD, respectively. The second panel (B) shows how each

Figure 2. Segmented raw vertical EOG data of a rejected trial showing both an eye movement

Figure 3. Representation of the distribution of the electrodes on the scalp and of the 
1 Figure 4. Potentials evoked in the frontal region of the scalp (Fz electrode) by parafoveal 2 orthographic distractors (RODs, dotted line) and their controls (UODs, solid line) in the literal task and the categorical task as a function of the nature of the foveal stimuli (hash symbols, a

4 UND, or another instance of the same type of distractor as the parafoveal word, i.e., a "repeated distractor"). The smaller frames displayed on some of the panels show the anatomical distribution on the scalp of the significant differences that were found between the P2a (140-220 ms) and N300 (260-320 ms) components evoked by RODs and UODs.

9 Figure 5. Potentials evoked in the frontal region of the scalp (Fz electrode) by parafoveal semantic distractors (RSDs, dotted line) and their controls (USDs, solid line) in the literal task

11 and the categorical task as a function of the nature of the foveal stimuli (hash symbols, a 12 UND, or a "repeated distractor"). The smaller frames displayed on some of the panels show the anatomical distribution on the scalp of significant differences that were found between the P2a component (140-220 ms) evoked by RSDs and USDs.

Figure 6. Potentials evoked by the critical search displays in the centro-parietal region of the scalp (CPz electrode) as a function of the nature of the foveal stimuli (hash symbols, a UND, or a "repeated distractor") on the left panel, and as a function of the nature of the search task (literal or categorical) on the right panel. The frames displayed on each panel show the anatomical distribution on the scalp of significant differences that were found for the N400 (450-550 ms) component evoked by different types of search displays. 